\title{
Nuclear and radiological emergency management and preparedness
}

\author{
Federico Rocchi ${ }^{1, *}$, Isabelle Devol-Brown ${ }^{2}$, and Wolfgang Raskob ${ }^{3}$ \\ ${ }^{1}$ FSN-SICNUC-SIN, ENEA, CR ENEA Bologna, Via Martiri di Monte Sole 4, 40129 Bologna, Italy \\ 2 PSE-SANTE/SESUC, IRSN, B.P. 17, 92262 Fontenay-aux-Roses Cedex, France \\ ${ }^{3}$ IKET CEDIM, KIT, Hermann-von-Helmholtz Platz 1, 76344 Eggenstein-Leopoldshafen, Germany
}

Received: 12 March 2019 / Accepted: 4 June 2019

\begin{abstract}
Recent EURATOM research efforts on Emergency Preparedness and Response (EP\&R) have been focussed on programs addressing some main knowledge gaps clearly identified in the outcomes of investigations carried out in Europe in response to the Fukushima accident. The PREPARE and FASTNET projects tried to solve similar problems adopting very complementary and synergic approaches. The main achievements of both projects are detailed in this paper. In particular, the problem of the fast estimation of time-dependent, longlasting Source Terms is discussed. This problem is not only a technical one, but is also related to the experience and skill of the code users. As the EP\&R is spanning a wide range in Europe, certainly far beyond the borders of individual states, it is mandatory creating a common and shared understanding of emergencies. Both PREPARE and FASTNET recognized the fundamental role of exercises to increase the experience of emergency responders in Europe. A general recommendation can then be formulated, in that more efforts should be dedicated in the future to the realization of such important exercises.
\end{abstract}

\section{Introduction}

Research and Development in the area of Nuclear and Radiological Emergency Management and Preparedness under the EURATOM umbrella went on in the last years with two major projects, namely, the PREPARE Collaborative Project (from 1 February 2013 to 31 January 2016, coordinated by KIT) $[1,2]$ and the FASTNET Research and Innovation Action (from 1 October 2015 to 30 September 2019, coordinated by IRSN) [3,4]. The first project was funded through the FP7-Euratom program and the second through the H2020-Euratom program. Both projects aimed at improving the existing Emergency Preparedness and Response (EP\&R) in Europe, and at addressing and closing some important gaps identified during, and in the aftermath of, the Fukushima Daiichi accident [5]. The outcomes of the analysis of the European reaction to the Japanese accident showed several important and common issues, which can be summarized as follows:

- missing early and rapid information on the potential Source Term (ST);

- absence of coordinated plan at European level to estimate the ST;

\footnotetext{
* e-mail: federico.rocchi@enea.it
}

- absence of a harmonized response to the safety of the European residents living in Japan;

- partly chaotic communication with the public;

- insufficient guidance on how to deal with incoming goods from Japan.

Both projects tried to tackle these points addressing them from different perspectives, adopting different methods, with synergic and highly complementary approaches, avoiding any duplication of efforts, as well. Both projects gave their own contributions to the solution of the extremely complex problem of enhancing the coherence in the preparedness and response to a nuclear emergency for a continent, Europe, which is very dense both in population and in nuclear power installations, and - at the same time - very diversified and heterogeneous as far as the nuclear technologies, the national legal frameworks, and the orography are concerned. The fast and timely delivery of comprehensive information about an existing or developing future situation is certainly a key point for decision-making in the early stage of an emergency. Fast and reliable ST assessments, not necessarily of a strongly conservative nature, are at the very heart of the problem. In this regard, PREPARE included among its goals the initial development and implementation of tools to derive a ST, using inversion algorithms as well as real-time ambient gamma dose-rates measured at 
the boundary of NPPs. To achieve the same goal, FASTNET developed fast-running tools to predict STs using a minimum set of plant data. Both approaches are valid and both need to be implemented and further strengthened; nevertheless, in case of missing dose-rate data, only the second approach can work, while dose-rate data may help correct calculated STs if wrong assumptions have been made in the calculations themselves. An example of synergy between the two approaches could be found in the fact that source inversion algorithms need a first-guess ST, the accuracy of which impacts directly the effectiveness of the inversion; this first-guess Source can be obtained with the tools developed in FASTNET. The complementarity between the two working methods shows-up in situations which require an early prognosis of an emergency, in order to timely activate and trigger protective countermeasures; such a prognosis must be made prior to any release to the environment, and therefore before the availability of any measured data; this prognosis is therefore enabled by fast-running tools. Measured data can be used, later on, either to confirm or to improve the calculated prognosis.

Both projects have got the common goal of achieving a more harmonized interpretation of an emergency situation, and therefore supporting more coherent decisions on protective actions to be implemented; that is mandatory to strengthen the confidence of the public in the safe use of nuclear power. Again, both projects contributed to this goal in different but synergic manners. PREPARE tried to foster analytic skills, providing a better guidance on how to communicate with the public and other stakeholders. FASTNET improved and, most importantly, shared among the stakeholders a common methodology for diagnosis and prognosis of emergencies and for the fast estimate of STs.

A third example of the complementarity and synergy of the two projects is the emphasis and efforts that both projects devoted to long-lasting radioactive releases. One of the lessons-learned from the Fukushima Daiichi accident is that a release of contaminants can be unevenly spread in time over several days, if not weeks. That was something rather unexpected and surely unprecedented, and immediately triggered reactions in the $\mathrm{EP} \& \mathrm{R}$ community worldwide to support the development and release of codes and tools, both for ST estimate and for atmospheric dispersion, able of dealing with such long lasting situations. For example, the US-NRC asked for improvements in the RASCAL fast-running code, the range of which, prior to Fukushima, was limited, in time, to $48 \mathrm{~h}$ of release and atmospheric transport and, in space, to $80 \mathrm{~km}$ distance from the source, only. Now its operation domain has been extended to $96 \mathrm{~h}$ and $160 \mathrm{~km}$ distances. PREPARE tackled this issue through a stress test-like simulation of the existing national operational procedures in Europe to verify their compliance to scenarios with very long-lasting releases. FASTNET, on the contrary, focused on the development of tools able to deal with situations up to a couple of weeks long-lasting, and even more, and to increase the awareness of the users of the tools in the fact that the time-dependence of a release is of the utmost importance to set-up properly protective countermeasures.
Detailed descriptions of the main findings and results of both projects will be given in the next sections.

\section{The PREPARE project}

This project started in February 2013 and ended in January 2016; it gathered 45 partners from Europe and the Fukushima University from Japan. The activities have been performed in seven work packages, with the following main aims and achievements:

- Operational procedures for long lasting releases: following the Fukushima Daiichi accident, a review of existing EP\&R procedures for long lasting releases and identification of possible needs for improvements by performing scenario calculations has been performed at a European level. Suggestions for improvements have been formulated.

- Platform for information collection and exchange: the socalled Analytical Platform for information exchange in time of nuclear or radiological crisis has been created. It allows discussion between institutional and non-institutional experts on an expert-level, and spreads congruent information on the current situation to the public, including mass media.

- Management of contaminated goods: stakeholder panels have been prepared, and panels had meetings in 10 European countries to review existing guidance and to identify areas for improvement.

- Improvement of decision support systems: the atmospheric dispersion models implemented in the two Decision Support Systems (DSS) ARGOS [6] and RODOS [7], as well as the hydrological model chain of RODOS, were extended. Among others, two methods for source-term estimation were developed and implemented. The long-term watershed model MOIRA was integrated, and the global ocean model MyOcean was linked to RODOS allowing using the simulations of this model as boundary conditions for the simulation of radionuclide dispersion in RODOS.

- Communication with the public: the overall objective of the work package was to investigate the conditions and means for relevant, reliable and trustworthy information to be made available to the public. Here, both traditional and social media were studied.

- Training, exercises and dissemination: training and exercising was an important aspect and therefore treated as a separate work package.

Concerning the operational procedures for long lasting releases, PREPARE made a stress test-like simulation to verify compliance with ICRP reference levels. In all countries with nuclear installations, detailed emergency management strategies have been developed in the past. In nearly all cases, such strategies are based on accident scenarios where the duration of the release of radionuclides to the environment is limited to either some hours or a few days at maximum. The Fukushima accident has demonstrated the likelihood of long lasting releases of radionuclides from an NPP over several weeks. That made it necessary to check the current off-site nuclear emergency 

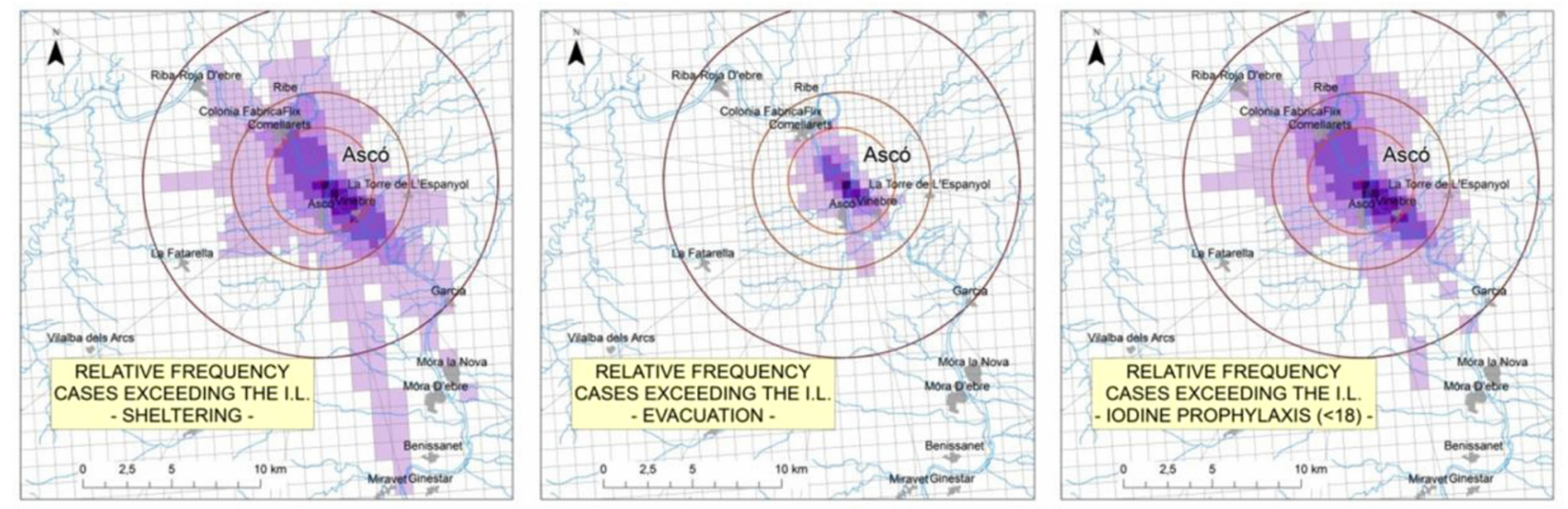

Fig. 1. Frequency of scenarios exceeding Spanish intervention limits.

plans in European countries against accident scenarios based on lessons learned from the Fukushima accident, and to derive recommendations on how to improve them. The tests should enable verifying whether protective measures foreseen in the current emergency plans could adequately reduce the radiological consequences of NPP accidents with long-lasting releases, similar to those from the Fukushima-Daiichi NPP. The methodology adopted consisted in identifying 10 representative STs, with duration of releases ranging from 22 to $188 \mathrm{~h}$. Seven of these tests can be classified at the INES ${ }^{1} 7$ level, two at the INES 6 and one at the INES 5. The total amount of ${ }^{131} \mathrm{I}$ released varied between 3 and $600 \mathrm{PBq}$. These scenarios were then combined with different atmospheric conditions (between 46 and 365 cases) to calculate doses to the population around several NPPs and the radiological consequences were compared to those assumed in the emergency planning. Areas and distances where national intervention criteria were exceeded have been identified; an example of such a case is given in Figure 1.

As a comprehensive result, it can be claimed that in a majority of release scenarios the areas calculated for protective actions do not exceed current planning zones. Were these ranges exceeded, the amount of affected population remains quite small. The number of sectors affected clearly increases with the duration of the release. If the release duration is lower than $12 \mathrm{~h}$, the affected sector is limited to less than $90^{\circ}$ in most cases. For very long lasting releases, however, the whole circular area (around the release point) could be affected (up to $360^{\circ}$ ).

The current intervention criteria in all countries guarantee that the residual dose in the first year (ICRP reference level) does not exceed $100 \mathrm{mSv}$. Even if the general findings of the project support the current planning, some shortcomings were identified, such as, for long lasting severe releases, a one-time intake of stable

\footnotetext{
${ }^{1}$ International Nuclear and Radiological Event Scale, maintained by IAEA and developed to classify STs according to their severity for people and the environment; currently the INES scale goes from level 1 (least severe) to level 7 (https://www.iaea.org/ publications/10508/ines-the-international-nuclear-and-radiologi cal-event-scale-users-manual).
}

iodine is often not sufficient for protecting the population against large thyroid doses. Multiple intakes of stable iodine tablets may have not been sufficiently considered in emergency planning.

As a side activity, also the consequences with respect to drinking water were investigated. The following findings can be reported:

- in case of a nuclear accident, surface water can be contaminated by large amounts of radionuclides and may not be suitable for drinking water production;

- advanced treatment processes as ion-exchange and reversed osmosis do remove radionuclides effectively, but these processes are not common practice;

- soil passage (dune infiltration, river bank filtration, groundwater) is a safe barrier for I-131 and Cs-137;

- if surface water is the main direct source for drinking water production, emergency plans for drinkable water supply are needed;

- drinking water utilities in the European countries are required by the EU Drinking Water Directive to provide emergency drinking water in case of a major accident, including nuclear accidents.

Two important open questions remain unanswered: - A long lasting, low release rate, atmospheric discharge would probably require a very large capacity in air sampling monitoring to achieve good measurements; have these special and non-standard monitoring devices ever been considered in the emergency plans and then put into operation?

- Is the evacuation of the population during the passage of the plume nearby always preferable against sheltering?

This second question refers to the fact that a choice is to be made quickly, either to evacuate or to order sheltering, during the passage of a plume. Typically, evacuation is recommended in the current emergency plans; however, it appears that in many cases sheltering is preferable because of the uncertainties in the ST and weather conditions, which may cause an erroneous choice of the evacuation routes. In this regard, the recently amended EU Safety Directive (article 8a(a), [8]) asks that safety arrangements are to be made in order to avoid "early radioactive releases that would require off-site emergency measures but with 
insufficient time to implement them." In principle, then, evacuation can still be implemented, but there should always be enough time to implement it safely.

Concerning contaminated foodstuff and feedstuff, in the framework of PREPARE an inter-comparison among 10 countries was made. An open discussion on the findings was launched, involving also EC, FAO, OECD-NEA, IAEA, HERCA and ICRP.

As far as improvements introduced in European DSSs, these were concentrated by PREPARE on ARGOS and RODOS. In particular, in the field of atmospheric transport, five different particle size classes were introduced in the dispersion models. They comprise small particles, around $1 \mu \mathrm{m}$ in diameter, up to heavy particles, about $60 \mu \mathrm{m}$ in diameter. As a boundary condition, the gravitational settling velocity for particles larger than $10 \mu \mathrm{m}$ will dominate the deposition process. These 5 different particle sizes were introduced in the dispersion models of the two decision support systems, and a corresponding deposition scheme developed and implemented. Additionally, two approaches of ST estimation, based on measurements and atmospheric dispersion models, were developed:

- a simple and fast technique that uses very simple dispersion modelling and gamma dose rate measurements in the near vicinity of a NPP;

- a more advanced technique that uses either detailed dispersion modelling and gamma dose rate or other measurements also at larger distance from the NPP.

The numerical methods are based on the sourcereceptor matrix (SRM), a linear regression technique. Prior information about the ST, the so-called first guess ST, is needed to regularize the linear regression and to assure uniqueness of the solution. The issue of unknown nuclide composition of the release has been handled enlarging the SRM and measurement vector using the ratios of release rates calculated through the first guess ST. The parameters of the regression include error variances of the first guess ST, error variances of observations, of simulated results and of nuclide ratios used in the enlarged minimization problem. Both tools, however, need more robust implementation and some activity on this has also been planned within the FASTNET project. Testing of the inversion algorithms has been performed using artificially generated "measurements" obtained for the meteorological and geographical conditions of the well-known ETEX experiment. Results of the test are reported in Figure 2.

Concerning the transport of radionuclides in water, the aim was to extend the Hydrological Dispersion Module of RODOS (RODOS-HDM), incorporating in it also the MOIRA DSS. MOIRA is a DSS created for the management of fresh water ecosystems contaminated by radionuclides and heavy metals [9].

An important issue for PREPARE, which is also of interest for FASTNET, is training and exercising. Two exercise sessions were organised, one focused on radiological assessment, supported by the use of JRODOS, and the other consisting of a more extensive table-top exercise with a simulated accident scenario. In addition, two table-top exercises were organized, dealing with a transport accident

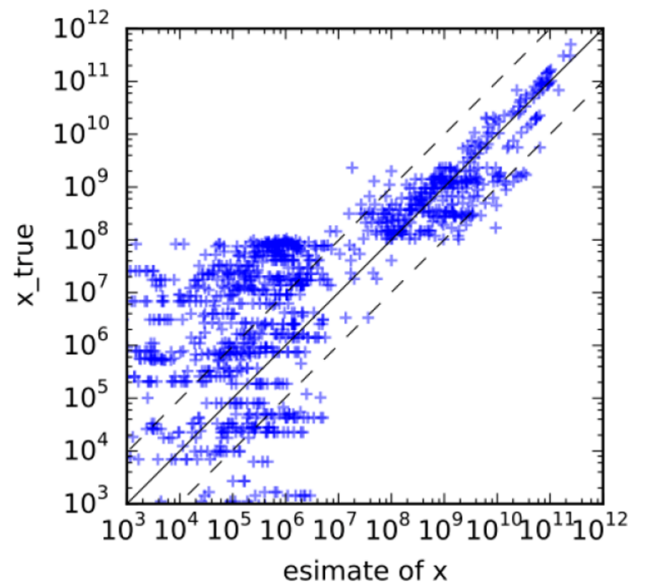

Fig. 2. Comparison of "true" and "estimated" ETEX source strength.

and with monitoring of a large scale cross-border contamination, respectively.

In summary, it can be said that the PREPARE project was successful in many aspects, as it dealt with some of the main gaps in EP\&R, which were found from the Fukushima experience. It created much more awareness in the strength and robustness on one side, and in the weak points on the other, of current emergency plans, as far as long-lasting releases are concerned. It also paved the way to the development of inverse methods to estimate STs from measurements and their implementation in the main European DSSs.

\section{The FASTNET project}

The FASTNET (Fast Nuclear Emergency Tools) project started in October 2015 and is expected to end in September 2019. It gathers 20 partners, coordinated by IRSN, together with IAEA. The aims of FASTNET are centred on three major pillars:

- the development of a reference SA scenarios database

[10], inclusive of time-dependent, isotopic STs, created using best-estimate SA codes (ASTEC [11], MAAP [12] and MELCOR [13]);

- the extension of existing methods (3D3P) and fastrunning codes (PERSAN and RASTEP) to predict STs to all current nuclear power plant technologies deployed in Europe (PWR, BWR, VVER, CANDU, EPR, including a generic model for SFPs) and their further development;

- the dissemination of best-practices on the use of the methods and tools developed within the project to estimate STs in real-time and during conditions typical of real emergencies.

During a real case of emergency, the time to perform ST calculations is undoubtedly very limited, as it is limited the amount and precision of the available information and plant data from the affected NPP. Therefore, best-estimate codes cannot be used to address the needs of a nuclear 


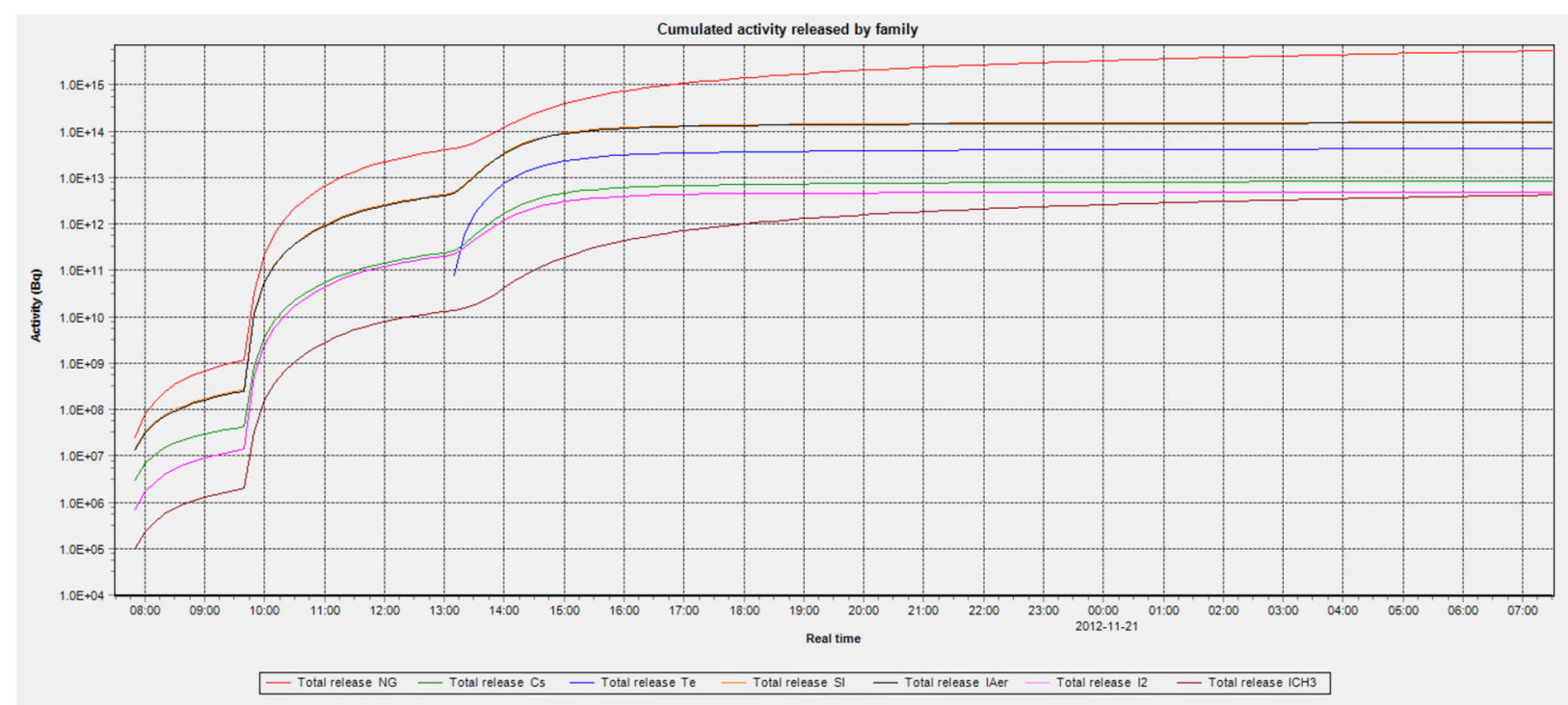

Fig. 3. Example of time-dependent ST obtained with PERSAN.

emergency; fast-running codes need instead to be developed and, most importantly, experience in their efficient and effective use must be built and spread out. As evidenced by the outcomes of the OECD-NEA FASTRUN project [14], which actually prompted and urged the creation of the FASTNET project, the knowledge and experience in Europe in the use of fast-running tools for ST prediction is, actually speaking, at best, very limited. Surely, not enough to serve the purpose of getting a shared and common vision of the accident progression and its consequences in terms of releases to the atmosphere. To address this major challenge, it was suggested within FASTNET to improve and disseminate a methodology for the diagnosis of plant status and for the prognosis of accident sequence, the 3D/3P (developed by IRSN), as well as two European fast-running tools, the French code PERSAN (developed by IRSN) and the Swedish code RASTEP (developed by LR), by extending their capabilities to all European NPP technologies, and to start to disseminate best practices in their use.

The 3D/3P, acronym for Triple Diagnosis/Triple Prognosis, is an analytical method which enables providing a simplified quick diagnosis of plant condition and the prognosis of a postulated future situation, evaluating the status and integrity of the typical three barriers of the defence-in-depth: fuel and cladding, primary circuit, and reactor containment. The method consists in filling a matrix composed of simple assessment judgements on the safety functions associated to the three barriers, namely: subcriticality and primary liquid inventory for the integrity of the first barrier, heat removal from primary system and from pump seals for the second barrier, and heat removal from the containment for the third barrier. The judgements are made both for the current situation and for a prognosis for the future. This method has been developed by IRSN for PWRs and within FASTNET it has been extended to other reactor types, including BWR, current VVERs, CANDU and SFPs. For CANDU technology the method has been renamed $4 \mathrm{D} / 4 \mathrm{P}$, given the peculiar nature of the safety barriers of these reactors, which include also the calandria vessel in series with the containment.

PERSAN is a deterministic code able to evaluate timedependent STs in a time-frame of a few minutes, provided that some realistic assumptions, such as either the timing of core dewatering or the specific leak-rates to the environment are given as input (see Fig. 3). The calculation methods are based on the solution of balance equations for radioisotopes defined over several volumes, in which the NPP is subdivided, schematized as lumped parameters, the imposed leak-rates serving either as boundary conditions to the atmosphere or as a link between the lumped parameters. Removal of radioisotopes from the volumes is based on conservation laws and on physical mechanisms like dry or wet (i.e. through the activation of spray systems) deposition, leak, filtering, radioactive decay, etc. All physical pathways to the atmosphere are considered, as a combination of parallel or series of flows. Chemical phenomena are modelled by very simple correlations. Initial core inventories are provided. Like for $3 \mathrm{D} / 3 \mathrm{P}$, PERSAN had been developed by IRSN for PWRs, and within FASTNET it has been extended to other reactor types, including BWR, VVER, CANDU and SFPs.

RASTEP [15] is a probabilistic code which can select, among a set of several pre-calculated STs for a given reactor type, the $\mathrm{ST}$ with the highest probability of occurrence for given plant conditions. The code is made of three main components: a Graphical User Interface (GUI, see Fig. 4), a Bayesian Belief Network (BBN) for each reactor type, and a database of pre-calculated accident sequences with related STs. These can be either obtained from the outcomes of PSA-2 studies of a given, real plant, or calculated ad-hoc with SA codes using generic plant schemes. Aim of the BBN is to connect partially available plant status data to one or more possible and compatible end states, represented by given STs, like in faulttree analyses. Introducing some plant conditions, some 


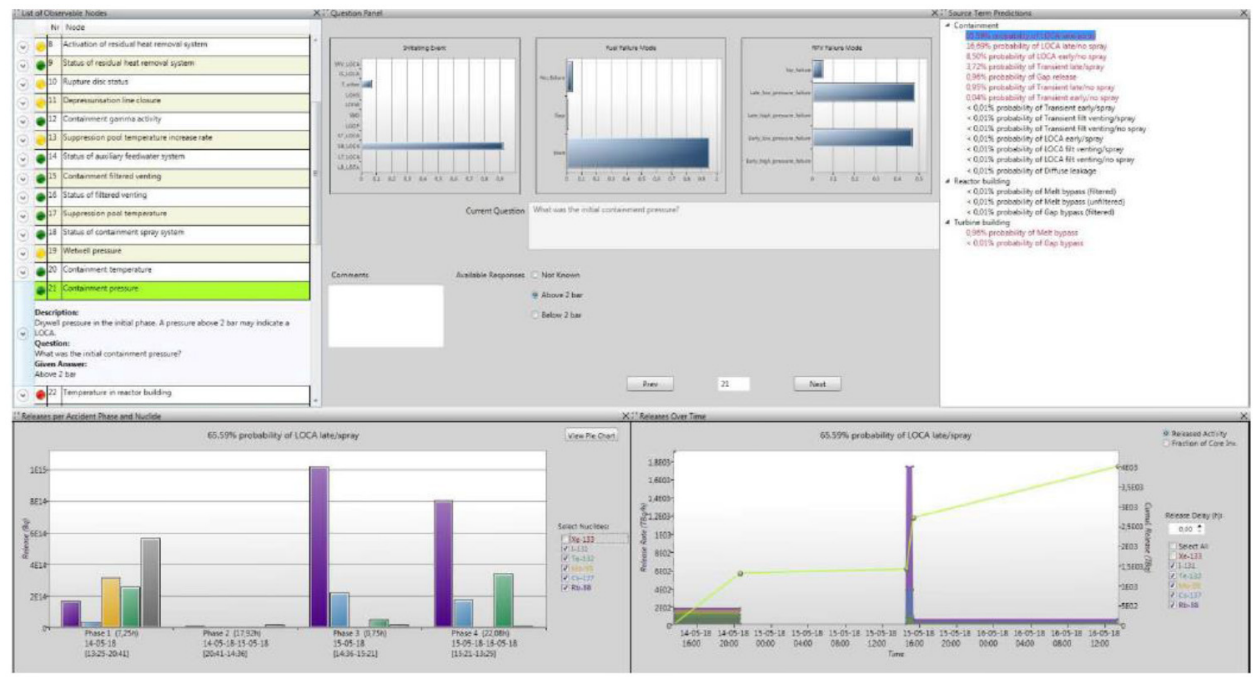

Fig. 4. RASTEP Graphical User Interface.

branches of the BBN are either isolated and further excluded from the analysis, or kept "open" and navigated up to an end state (or states) with associated conditional probability or likelihood of occurrence. The more information on the plant status is provided by the user, the higher is the probability of reaching a good ST for the situation under scrutiny. The approach is clearly based on the assumptions of having a sufficiently large database of sequences to cover the most of the accidental situations and a robust BBN to map correctly the database. The GUI is used to provide information to the code by answering a limited set (some tens) of simple questions on the safety barriers and safety safeguards. Their availability or unavailability determines which boughs of the tree are to be selected and, in case of more than one final plant status, which probabilities can be associated with the different results. RASTEP has been initially developed by LR for SSM for BWR and within FASTNET its use has been expanded to include also PWR, VVER and CANDU. The extension consisted in the creation of dedicated BBNs for each reactor type as well as the ad-hoc database of reference STs.

To fill the RASTEP databases, another goal of FASTNET was the development of a comprehensive database of reference STs, calculated with best-estimate codes like ASTEC, MAAP and MELCOR, for as many sequences as possible: a huge effort indeed for the partners because were not the STs already available, they had to be evaluated from scratch. The reference STs had obviously to be given in terms of time-dependent isotopic releases, which was really challenging for partners using those SA codes, which only deal with chemical classes. The database is also a set of reference scenarios against which it is possible to test and validate the behaviour of the fastrunning codes. This implies that they should contain not only data for the temporal progression of the accident sequences and time-dependent STs, but also many thermal-hydraulic time-dependent data on the primary circuit, as well as physical data on the containment status.
Accordingly, given the precious nature of the information contained in the FASTNET database, it was decided to transfer it to the IEC of IAEA for the purposes of maintenance in time, beyond the lifetime of FASTNET, and for further future expansion. IAEA CPs would be allowed, in principle, to search the database on-demand, in case of specific needs (including training), or during real emergencies, which might be similar to a scenario already available in the database. The development of the FASTNET database proved to be a very ambitious, time-consuming, and highly demanding task. Two problems are still pending concerning the sequences currently available: the number of sequences itself, and the quality control of their data. As of today, the database comprises about 120 sequences, and a few more are planned to be added before the end of the project. Despite this big number, the database is far from being complete and exhaustive, and many more years of work should be needed to reach a level, which can be considered more or less satisfactory for EP\&R needs. However, on one side it confirms the need of having fast-running codes, on the other cannot be seen as an excuse to limit the use of bestestimate codes for general EP\&R needs. Concerning the quality control of the provided STs, this was obviously beyond the scope and the limited resources of FASTNET, and therefore the FASTNET database is to be considered for now "as is". A further and final aim of the FASTNET database was to provide data for the preparation of another extremely important product of the project, namely, training in the form of emergency exercises.

To address the above-mentioned problem of training in EP\&R, the FASTNET project envisaged a twofold approach. On one side a one-week training on $3 \mathrm{D} / 3 \mathrm{P}$, PERSAN and RASTEP has been organized, during which the participants (not limited to project partners but open also to interested stakeholders forming the s.c. End Users Group) were trained on the practical use of the tools. On the other side, two exercises were organized. The first one consisted in the calculation of STs for four sequences 


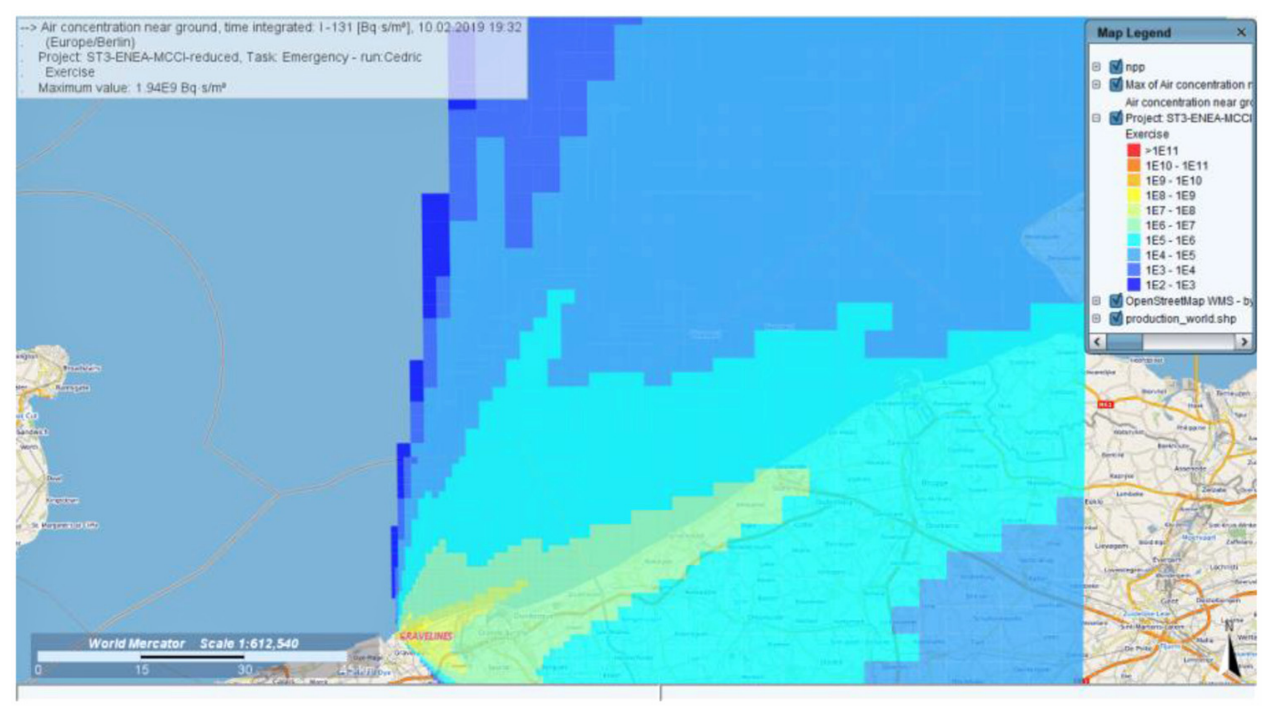

Fig. 5. Example of JRODOS dispersion calculation results from Exercise 2.

(a PWR, a BWR, a CANDU and a VVER) using both PERSAN and RASTEP. The aim of this exercise was to strengthen the user capabilities but it was also useful to acquire better confidence in the codes; therefore, time pressure was not given to participants and a full month was allocated to them to provide results. The outcomes of this exercise were manifold: further improvements of both PERSAN and RASTEP, and better consciousness of partners in their current knowledge and capabilities in using correctly fast-running tools. After this first exercise, targeted to ST estimation, a second was organized in the form of a real-time table-top exercise, during which partners had to calculate a ST for a given accidental situation and then provide, with their own atmospheric dispersion tools, estimates of the radiological consequences to the population. This exercise was very challenging, because of the time constraints, however proved to be enormously useful in getting more experience in the realtime use of the FASTNET products.

In the objective the STs can be used in different atmospheric dispersion codes and also shared among different emergency responders, they are to be standardized in terms of format of data and files. To address this requirement, a few years ago, IAEA developed the IRIX (International Radiological Information Exchange Format) [16], which is an xml-based information exchange format designed to facilitate web-based exchange of relevant emergency information and data among organisations that respond to nuclear and radiological incidents and emergencies, and in particular the exchange of emergency information among national authorities that have responsibilities assigned under the Convention on the Early Notification of a Nuclear Accident. An important byproduct of the FASTNET project has been the adoption in the fast-running tools, as well as in the exporting options of the database for the IAEA, of the IRIX format.

While still under development (the current version is 1.0), the IRIX format allows decoupling from an IT point of view ST calculation tools from atmospheric dispersion codes. Within FASTNET, IRIX output capabilities were introduced for PERSAN, RASTEP and the database, while input capabilities have been introduced in JRODOS. During the second exercise, some partners were therefore able to use PERSAN or RASTEP in conjunction with JRODOS thanks to the IRIX input/output functionalities (see Fig. 5). This is of course to be maintained for the future, since new and improved versions of the IRIX standard may be foreseen in the incoming years.

While still ongoing, it can already be stated that FASTNET has been a successful first opportunity to establish a link and a dialogue between the communities of scientists devoted to the best-estimate evaluation of STs, and that of those scientists using STs in their daily work of protecting people and the environment. Moreover, the development and the sharing of fast-running tools for STs and of associated, common, working methodologies is to be welcomed, as a first step in filling the most important gap in $\mathrm{EP} \& \mathrm{R}$, that of the fast, timely and accurate predictions of releases to the atmosphere.

\section{Dissemination and education and training}

Both projects dedicated resources and efforts to dissemination and education and training. These are important aspects of European projects, because they are the most effective way of sharing the knowledge gains, and to preserve them in time beyond the lifespan of the projects themselves. Both projects gathered end-user communities, which could directly benefit from the scientific results; these communities were invited to events and were given the opportunity to test the products of the research and to give feedback. Both projects organized trainings, workshops, seminars, schools and international conferences. For example, FASTNET organized a one week-long training on PERSAN and RASTEP in Paris in 2018. A one week-long, international School on EP\&R took place in Bologna in January 2019, with lectures also on PERSAN and 
RASTEP. The School was attended by several $\mathrm{PhD}$ students. Two international workshops have taken place within FASTNET, and a final one is going to be organized as a joint Side-Event by France, Sweden and Italy at the next IAEA General Conference. IAEA has been invited to the various FASTNET scientific events and some partners presented the project and its achievements at various meetings in Europe (f.i. NKS workshops) and USA (USNRC CSARP meetings). An important presentation was given on FASTNET at the 2017 ECURIE Competent Authorities meeting. PREPARE organized a dissemination workshop in Bratislava in 2016, and several presentations were given at the NERIS workshop in 2015 in Milan. It also organized two basic courses on emergency management and rehabilitation. The first course (2014) focused on the early to intermediate phases after a nuclear/ radiological accident, whereas the second course (2015) was related to the long term management of contaminated territories. Finally, a training course on the PREPARE Analytical Platform was organized in Trnava in 2015. The aspect of financing Master degree theses, $\mathrm{PhD}$ or post-doc positions was considered by both projects, however, it resulted very difficult to find candidates, given also the fact that (a) these three figures (Masters, PhDs and post-docs) can, by law, be dealt with only by universities and not by research entities, and that (b) the costs to fund these positions vary enormously from country to country. These two drawbacks and limitations should be better considered by the European Commission, for example with special funding rules, if in the future more efforts are to be devoted to direct higher education actions.

\section{Conclusions}

Both PREPARE and FASTNET projects tried to close some gaps identified in EP\&R capabilities in Europe; they both tried to implement in practice some lessons learned from the Fukushima Daiichi accident. Both gave complementary contributions to solve fundamental problems of EP\&R. Much has been done, as detailed in the previous paragraphs, but much still needs to be achieved.

For example, one major challenge, which was anticipated and actually experienced in the FASTNET project, is related to the dialogue between the severe accident management scientific community and the emergency management one. These two communities have the same final aim of protecting people through increase in safety; they, however, speak different languages and are used to tackle similar problems but with different perspectives. FASTNET was the first European project on EP\&R in which these two communities were gathered together and were asked to cooperate; there was then an additional operational aim within FASTNET: to find a common language, harmonize the practices, use the tools that are most relevant and easy to use for them, and facilitate their appropriation of the common methodology proposed. This first dialogue attempt was certainly fruitful, but not complete. In the future it is then highly recommended that opportunity is given to strengthen the developed links between these two communities, for example by organizing
(1) operational trainings based on every technology and the feedback from the exercises organized within FASTNET; (2) a new series of exercises, targeting the protection of population and having a higher level of reality (full-scale formats, scenarios based on every technology, etc.). It must in fact be recognized that much more training is needed on the fast-running tools developed, especially in their use in emergency centres. As evidenced for the PREPARE project and as already introduced before about the outcome of the FASTRUN OECD/NEA project, training in EP\&R is really an absolute need for Europe. The development of fast-running codes is per se not enough if potential emergency responders are not properly trained in dealing with such tools and the phenomena they describe. Further development of the reference SA database is also necessary. The complementarity between the results of PREPARE and FASTNET should be taken to the level of productive interaction, for example by using STs derived from fast-running tools to aid the procedures of ST estimate from dose-rate measurements (inverse methods). This kind of interaction was also suggested by the NERIS gap analysis (Area 1, Key Topic 3) $[17,18]$ where it is explicitly stated "Link of inverse with in-plant (e.g. FASTNET project) ST estimation methodologies". Another important improvement for the future could be the development of uncertainty propagation using STs evaluated by fast-running tools and ensemble data from numerical weather predictions.

\section{References}

1. PREPARE, https://cordis.europa.eu/project/rcn/106584/ factsheet/en

2. PREPARE Final Report, PREPARE(WP8)-(16)-03, 2016

3. FASTNET, https://cordis.europa.eu/project/rcn/198668/ factsheet/en

4. I. Devol-Brown, F. Di Dedda, J.E. Dyve, O. Isnard, F. Rocchi, E. Urbonavicius, D. Vola, FASTNET: FAST Nuclear Emergency Tools, in ERMSAR 2017, Warsaw, Poland

5. R. Mustonen, C. Rojas-Palma, W. Raskob, EURATOM FP7 Projects on Management of Nuclear and Radiological Emergencies, in FISA 2013 Proceedings, Spain, 2014, pp. 261-268

6. S. Hoe, P. McGinnity, T. Charnock, F. Gering, L.H. Schou Jacobsen, J. Havskov Sørensen, P. Astrup, ARGOS Decision Support System for Emergency Management, in Proceedings of the Argentine Radiation Protection Society, 2009

7. A. Wengert et al., JRodos: an off-site emergency management system for nuclear accidents, KIT, 2017

8. Council Directive 2014/87/EURATOM of 8 July 2014

9. L. Monte, L. Håkanson, D. Hofman, J.E. Brittain, E. Gallego, A. Jiménez, G. Angeli, MOIRA-Plus: a decision support system for the management of complex fresh water ecosystems contaminated by radionuclides and heavy metals, Comput. Geosci. 35, 880 (2009)

10. F. Mascari et al., FASTNET Scenarios Database Development and Status, submitted to ERMSAR 2019, Prague, Czech Republic

11. P. Chatelard, S. Belon, L. Bosland, L. Carénini, O. Coindreau, F. Cousin, C. Marchetto, H. Nowack, L. Piar, L. Chailan, Main modelling features of ASTEC V2.1 major version, Ann. Nucl. Energy 93, 83 (2016) 
12. Fauske \& Associates, LLC, Transmittal document for MAAP5 code revision MAAP 5.02, FAI/13-0801, 2013

13. US NRC, MELCOR Computer Code Manuals, Vol. 1: Primer and Users' Guide, SAND 2015-6691 R; Vol. 2: Reference Manual, SAND 2015-6692 R; Vol. 3: MELCOR Assessment Problems, SAND 2015-6693 R (Sandia National Laboratories, USA, 2015)

14. Benchmarking of Fast-running Software Tools Used to Model Releases during Nuclear Accidents, Report NEA/ CSNI/R(2015) 19
15. F. Di Dedda, A. Olsson, J. Klug, A. Riber Marklund, RASTEP: a novel tool for nuclear accident diagnosis and source term prediction based on PSA and Bayesian Belief Networks, LR Nucl. Tech. Dig. 1, 14 (2018)

16. International Radiological Information Exchange (IRIX) Format, Version 1.0, IAEA Incident and Emergency Centre, 2013

17. Research needs identified from the NERIS gap analysis, October 2018

18. Strategic Research Agenda of the NERIS Platform, September 2017

Cite this article as: Federico Rocchi, Isabelle Devol-Brown, Wolfgang Raskob, Nuclear and radiological emergency management and preparedness, EPJ Nuclear Sci. Technol. 6, 37 (2020) 